\title{
Detecting a Network Failure
}

\author{
Jon Kleinberg
}

Abstract. Measuring the properties of a large, unstructured network can be difficult: One may not have full knowledge of the network topology, and detailed global measurements may be infeasible. A valuable approach to such problems is to take measurements from selected locations within the network and then aggregate them to infer large-scale properties. One sees this notion applied in settings that range from Internet topology discovery tools to remote software agents that estimate the download times of popular web pages. Some of the most basic questions about this type of approach, however, are largely unresolved at an analytical level. How reliable are the results? How much does the choice of measurement locations affect the aggregate information one infers about the network?

We describe algorithms that yield provable guarantees for a particular problem of this type: detecting a network failure. Suppose we want to detect events of the following form in an $n$-node network: An adversary destroys up to $k$ nodes or edges, after which two subsets of the nodes, each of size at least $\varepsilon n$, are disconnected from one another. We call such an event an $(\varepsilon, k)$-partition. One method for detecting such events would be to place "agents" at a set $D$ of nodes, and record a fault whenever two of them become separated from each other. To be a good detection set, $D$ should become disconnected whenever there is an $(\varepsilon, k)$-partition; in this way, it "witnesses" all such events.

We show that every graph has a detection set of size polynomial in $k$ and $\varepsilon^{-1}$, and independent of the size of the graph itself. Moreover, random sampling provides an effective way to construct such a set. Our analysis establishes a connection between graph separators and the notion of VC-dimension, using techniques based on matchings and disjoint paths.

(C) A K Peters, Ltd.

|542-795|/03 $\$ 0.50$ per page 


\section{Introduction}

\section{I.I. Inferring Network Properties}

In large, unstructured networks, it can be difficult to answer even the most basic questions-What does the topology look like? How many (large) connected components are there? What is the average distance (or packet roundtrip time) between pairs of nodes? Indeed, a vein of recent work has focused on the issue of designing algorithms that operate on networks for which we do not necessarily have an explicit representation, or for which detailed global measurements are not feasible (e.g., [Chung et al. 01, Claffy et al. 99, Paxson 96, Ratnasamy and McCanne 99, Siamwalla et al. 98] and, in a fairly different context, [Bharat and Broder 98, Lawrence and Giles 99]).

Such work has arisen from a variety of different motivations, but much of it - especially in the context of Internet performance metrics - is based on the notion of taking measurements from selected locations within the network, and then aggregating these measurements to infer large-scale network properties. This approach is used, for example, in structural analysis based on Internet tomography [Chung et al. 01, Claffy et al. 99], algorithms for discovering Internet topology [Siamwalla et al. 98] and routing structures [Ratnasamy and McCanne 99], and mechanisms for estimating a variety of Internet performance metrics [Paxson 96]. Companies such as Keynote Systems [Keynote 03] provide information on the average response time of home page requests to high-volume web sites by combining data collected from software "agents" distributed through the Internet.

This type of consideration - combining data from measurement agents in a network - forms the crux of our concerns in this paper. How does the choice of locations for such agents affect the aggregate information one infers about the network? How large a subset of locations do we need in order to achieve a desired level of confidence in such information? For most natural performance metrics, these questions lead quickly to open problems.

\subsection{Network Connectivity}

We formulate a concrete inference problem concerning one of the most fundamental network properties - connectivity. Consider an undirected graph $G$ on $n$ nodes, which is initially connected. Suppose, for a parameter $\varepsilon>0$, we are interested in detecting $\varepsilon$-partitions: failures of network elements after which there are two subsets of nodes $A$ and $B$, each of size at least $\varepsilon n$, such that no node in $A$ has a path to any node in $B$. For a parameter $k>0$, we wish to be able to detect any $\varepsilon$-partition that is caused by the failure of up to $k$ (adversarially chosen) 
nodes or edges, and record the occurrence of such an event. Note that increasing $k$ allows an adversary more power, so handling larger values of $k$ represents a sequence of successively more difficult problems.

Here is a general approach for doing this, motivated by the type of analysis discussed above. We place "monitoring agents" at a subset $D$ of the nodes of $G$, and each of these agents periodically engages in communication with all the others. Now, if at some point in time, there are nodes $u, v \in D$ such that the agents at $u$ and $v$ have no path connecting them, we can record a fault in the network. Such a protocol clearly has the property that a fault is only recorded if the network has actually become disconnected; we would like to choose $D$ so that a fault is always recorded when the deletion of up to $k$ nodes or edges has resulted in an $\varepsilon$-partition. How large a set $D$ do we need in order to achieve this, for an underlying graph $G$ ?

We make this question precise as follows. Let $G=(V, E)$ be a connected graph; by an element of $G$, we mean one of its nodes or edges. We say that two sets of nodes $A$ and $B$ are separated if there is no path with one end in $A$ and the other in $B$. We say that a set of elements $Z$ is an $(\varepsilon, k)$-partitioning set if $|Z| \leq k$ and $G \backslash Z$ contain disjoint subsets of nodes $A$ and $B$, each of size at least $\varepsilon n$, that are separated. Finally, we say that a set $D \subseteq V$ is an $(\varepsilon, k)$-detection set if for every $(\varepsilon, k)$-partitioning set $Z$, there are two nodes $u, v \in D \backslash Z$ that lie in different connected components of $G \backslash Z$. Such a pair of nodes will no longer be able to communicate after the deletion of the elements in $Z$, and hence will "witness" the partitioning of the network.

Clearly, by taking $D$ to be the entire node set $V$, we obtain an $(\varepsilon, k)$-detection set. The question is whether there is a much smaller sample with the same property.

Our main result is that for every graph $G$, there is an $(\varepsilon, k)$-detection set whose size is bounded by a function of $\varepsilon$ and $k$ only, independent of the number of nodes. Specifically, we show

Theorem I.I. Every graph $G$ has an $(\varepsilon, k)$-detection set of size $O\left(k^{3} \varepsilon^{-1} \log \varepsilon^{-1}\right)$. Moreover, a set of

$$
O\left(k^{3} \varepsilon^{-1} \log \varepsilon^{-1}+\varepsilon^{-1} \log \delta^{-1}\right)
$$

nodes chosen uniformly at random is an $(\varepsilon, k)$-detection set with probability at least $1-\delta$.

Thus, the theorem also shows that uniform random sampling yields a detection set with high probability; in particular, this means that one can construct a small detection set without detailed local knowledge of the network topology. 
The heart of the proof is the analysis of this sampling procedure. For this, we use the notion of VC-dimension [Vapnik and Chervonenkis 71]; we define a particular set system over the vertex set of the graph, show that it has small VC-dimension, and then relate detection sets to $\varepsilon$-nets [Haussler and Welzl 87] for this set system. It turns out that the most natural set systems here actually have very large VC-dimension, and so are not useful in our analysis; formulating and analyzing a set system for which the VC-dimension is bounded raises some interesting graph-theoretic issues, including connections to theorems of Gallai and Mader on disjoint paths [Gallai 61, Mader 78] that generalize the TutteBerge theorem on nonbipartite matchings [Berge 58, Lovász and Plummer]. We view the connection developed here between VC-dimension and the separators of a graph as one of the contributions of this work, and believe that our style of analysis may be amenable to related problems as well.

The role of node and edge separators here suggests connections to problems such as approximating the failure probability of a network [Karger 95]. It is important to note, however, that the issues are quite different at a technical level. Specifically, we are concerned with choosing a fixed set of nodes $D$ from which we can detect any possible (adversarially chosen) failure of a particular type. Also, we allow here for node failures in addition to edge failures; network reliability allowing node failures, on the other hand, is largely an open question [Karger 95].

\section{I.3. Generalizations}

We will actually prove the following strengthening of Theorem 1.1. Suppose that the nodes of our graph are initially partitioned into two classes: end nodes $V_{0}$, and internal nodes $V_{1}$. We are only allowed to place monitoring agents at nodes in $V_{0}$, and we are interested in detecting $\varepsilon$-partitions of $V_{0}$.

Thus, we say that a set of elements $Z$ is an $(\varepsilon, k)$-partitioning set with respect to $V_{0}$ if $|Z| \leq k$ and $G \backslash Z$ contains disjoint subsets $A, B \subseteq V_{0}$, each of size at least $\varepsilon\left|V_{0}\right|$, that are separated. We say that a set $D \subseteq V_{0}$ is an $(\varepsilon, k)$-detection set for $V_{0}$ if for every set $Z$ that is $(\varepsilon, k)$-partitioning with respect to $V_{0}$, there are two nodes $u, v \in D \backslash Z$ that lie in different connected components of $G \backslash Z$. Our generalization of Theorem 1.1 is now simply the following.

Theorem I.2. For every graph $G=(V, E)$, and any set $V_{0} \subseteq V$, there is an $(\varepsilon, k)$ detection set for $V_{0}$ of size $O\left(k^{3} \varepsilon^{-1} \log \varepsilon^{-1}\right)$. Moreover, a set of

$$
O\left(k^{3} \varepsilon^{-1} \log \varepsilon^{-1}+\varepsilon^{-1} \log \delta^{-1}\right)
$$

nodes chosen uniformly at random from $V_{0}$ is an $(\varepsilon, k)$-detection set for $V_{0}$ with probability at least $1-\delta$. 


\section{I.4. Alternate Notions of Detection}

We note that there are two natural strengthenings of our notion of detection-in which we drop one or the other of the parameters $\varepsilon$ and $k$-but neither of these can be achieved in any nontrivial way.

Specifically, one natural strengthening would be to drop the parameter $\varepsilon$ and ask that a set $D^{\prime}$ have the following property: For any $Z \subseteq V \cup E$ of size at most $k$, if $G \backslash Z$ is not connected, then some two nodes of $D^{\prime} \backslash Z$ lie in different components of $G \backslash Z$. But if we consider any 2-connected $d$-regular graph, and set $k=d$, we see that $Z$ could consist of the $d$ edges incident to any node, and hence, we would need to take $D^{\prime}=V$.

A second natural strengthening would be to drop the parameter $k$ and ask that a set $D^{\prime \prime}$ have the following property: For any $Z \subseteq V \cup E$, if $G \backslash Z$ contains separated subsets $A$ and $B$, each of size at least $\varepsilon n$, then some two nodes of $D^{\prime \prime} \backslash Z$ lie in different components of $G \backslash Z$. But in this case, consider the star graph $G=K_{1, n-1}$, and suppose $D^{\prime \prime} \subseteq V$ has fewer than $(1-\varepsilon) n$ nodes. Let $Z$ consist of all edges incident to leaves of $G$ not in $D^{\prime \prime}$. $G \backslash Z$ contains two separated sets, each of size at least $\varepsilon n$, but $D^{\prime \prime}$ is completely contained in a single component of $G \backslash Z$.

\subsection{A Simple Bound Depending on the Size of the Graph}

From a qualitative perspective, the crucial feature of our main result is that the size of the detection set can be bounded independently of the size of the underlying graph. Indeed, if one is willing to accept a size bound depending on $n=|V|$ and $m=|E|$, then one can apply the following easy argument.

Suppose we select a set of nodes $D$ at random from $G$ to form our detection set, and let $c=|D|$. For any particular $(\varepsilon, k)$-partitioning set $Z$, the probability that $D$ lies entirely in a single component of $G \backslash Z$ is at most $(1-\varepsilon)^{c}$. If we choose $c=\Theta\left(k \varepsilon^{-1} \log (m+n)\right)$, this probability can be made less than $(m+n)^{-\beta k}$ for any constant $\beta>1$. But there are at most $(m+n)^{k}$ possible $(\varepsilon, k)$-partitioning sets (simply because there are at most this many ways to choose $k$ elements from $G$ ); hence applying the union bound over all such sets, we see that $D$ is an $(\varepsilon, k)$-detection set with high probability.

\section{I.6. Subsequent Results}

Since the appearance of the conference version of this paper, there have been two extensions of the results presented here. Fakcharoenphol [Fakcharoenphol 01] improved the VC-dimension bound we obtain for the set system defined in Section 3, leading to an improved bound of $O\left(k \varepsilon^{-1} \log k \log \varepsilon^{-1}\right)$ on the size of an $(\varepsilon, k)$-detection set in an arbitrary graph. 
Gupta [Gupta 00] introduced the notion of a weak detection set, which satisfies a less stringent requirement than our definition here; specifically, one says that $D^{\prime}$ is a weak detection set if for every $(\varepsilon, k)$-partitioning set $Z$, it is the case that $D^{\prime}$ intersects $Z$, or there are two nodes $u, v \in D^{\prime}$ that lie in different connected components of $G \backslash Z$. (In other words, as an alternative to having nodes of $D^{\prime}$ in different components, it is sufficient that the failure of $Z$ causes one of the nodes in $D^{\prime}$ to fail.) Gupta shows that every graph has a weak detection set of size $O\left(k \varepsilon^{-1}\right)$, a bound that is easily seen to be tight. His proof technique involves a construction in terms of the structure of the underlying graph, and does not imply a result for random sampling of nodes. Whether a comparable bound can be obtained under the definition of detection sets that we use here is an interesting open question.

\section{Detecting Edge Failures}

We begin by considering the special case in which only edges can fail. We also assume here that $V_{0}=V$. Focusing on this special case first provides a good illustration of the way in which we use VC-dimension arguments; it also allows us to work with a particularly natural set system defined over the vertex set for which we can show that our VC-dimension analysis is tight.

Thus, we say that $D \subseteq V$ is an $(\varepsilon, k)$-detection set with respect to edge failures if for every $(\varepsilon, k)$-partitioning set $Z$ consisting only of edges, some two nodes of $D$ lie in different components of $G \backslash Z$. We say that a set $S \subseteq V$ is $k$-edge-separable if there exists a set $Z$ of at most $k$ edges such that $S$ is the union of connected components of $G \backslash Z$.

It is not difficult to show that a set which meets every large $k$-edge-separable set is, in fact, a detection set.

Lemma 2.I. If $D \subseteq V$ intersects every $k$-edge-separable set of size at least $\varepsilon n$, then it is an $(\varepsilon, k)$-detection set with respect to edge failures.

Proof. Let $Z$ be a set of edges of size at most $k$, and suppose there exist disjoint sets $A, B \subseteq V$, each of size at least $\varepsilon n$, that are separated in $G \backslash Z$. Let $A^{\prime}$ denote the union of all components of $G \backslash Z$ that intersect $A$, and let $B^{\prime}$ denote the union of all components of $G \backslash Z$ that intersect $B$; note that $A^{\prime}$ and $B^{\prime}$ are disjoint. Also, each of $A^{\prime}$ and $B^{\prime}$ is a $k$-edge-separable set of size at least $\varepsilon n$; hence $D$ contains at least one node from each of $A^{\prime}$ and $B^{\prime}$, and these lie in different components of $G \backslash Z$.

We need the following background on the VC-dimension of set systems. Let $\Omega$ be a finite set and $\mathcal{X}$ a collection of subsets of $\Omega$. We say that $A \subseteq \Omega$ is shattered 
by $\mathcal{X}$ if for all $B \subseteq A$, there exists an $X \in \mathcal{X}$ such that $B=A \cap X$. The $V C$-dimension of the set system $(\Omega, \mathcal{X})$ is the maximum cardinality of a subset of $\Omega$ that is shattered by $\mathcal{X}$. Here is a simple lemma, which we note for later use.

Lemma 2.2. Let $(\Omega, \mathcal{X})$ be a set system, $\Omega^{\prime}$ a subset of $\Omega$, and $\mathcal{X} \mid \Omega^{\prime}=\left\{X \cap \Omega^{\prime}: X \in\right.$ $\mathcal{X}\}$. Then the $V C$-dimension of $\left(\Omega^{\prime}, \mathcal{X} \mid \Omega^{\prime}\right)$ is bounded above by the $V C$-dimension of $(\Omega, \mathcal{X})$.

We say that $N \subseteq \Omega$ is an $\varepsilon$-net with respect to $(\Omega, \mathcal{X})$ if for every $X \in \mathcal{X}$ of cardinality, at least $\varepsilon|\Omega|$, the set $N$ contains an element of $X$.

We now have the following theorem of Blumer et al. [Blumer 89], strengthening a result of Haussler and Welzl [Haussler and Welzl 87].

Theorem 2.3. ([Blumer 89, Haussler and Welzl 87]) There is a function

$$
f(d, \varepsilon, \delta)=O\left(d \varepsilon^{-1} \log \varepsilon^{-1}+\varepsilon^{-1} \log \delta^{-1}\right)
$$

such that the following holds. Let $(\Omega, \mathcal{X})$ be any set system with VC-dimension at most $d$. Then a random subset of $\Omega$ of size $f(d, \varepsilon, \delta)$ is an $\varepsilon$-net with probability at least $1-\delta$.

The crux of this theorem is the nonobvious point that there exist $\varepsilon$-nets for arbitrary set systems with a size that is polynomial in $\varepsilon^{-1}$ and the VC-dimension, and independent of the cardinality of the underlying set $\Omega$.

Taken together, Lemma 2.1 and Theorem 2.3 suggest that we focus on the set system $(V, \mathcal{S})$, where $\mathcal{S}$ consists of the $k$-edge-separable sets. Indeed, Lemma 2.1 states that an $\varepsilon$-net with respect to this set system will be an $(\varepsilon, k)$-detection set.

We bound the VC-dimension of this set system by the following line of reasoning: Any large set of nodes $A$ can be "paired up" in $G$ via edge-disjoint paths; deleting a set of $k$ edges cannot destroy all these paths; and hence there is no $k$-edge-separable set whose intersection with $A$ produces exactly one node from each group in our pairing. It then follows that $A$ cannot be shattered.

To show how such a pairing can be achieved, we use the following lemma.

Lemma 2.4. Let $H=\left(V_{H}, E_{H}\right)$ be a connected graph, and $T \subseteq V_{H}$ a set of terminals of cardinality $2 \ell$, for a natural number $\ell$. Then there exist mutually edge-disjoint paths $P_{1}, P_{2}, \ldots, P_{\ell}$ such that each node in $T$ appears as one end of exactly one of these paths. 
Proof. It clearly suffices to prove the result when $H$ is a tree; indeed, if it holds in trees, then for an arbitrary connected graph $K$, we can construct the desired disjoint paths in a spanning tree of $K$.

We will prove the result by induction on the number of nodes in $H$, with the two-node tree forming an easy base case. For a larger tree $H$, we observe that if any leaf $v$ does not belong to $T$, then we can delete $v$ and proceed inductively. Thus we will suppose that all leaves belong to $T$. Now, let $v \in T$ be an arbitrary leaf, let $w$ be the node adjacent to $v$, and consider the following two cases.

(i) $w \in T$. In this case, define $H^{\prime}$ to be the tree $H$ with edge $(v, w)$ and node $v$ deleted, and define $T^{\prime}=T \backslash\{v, w\}$. By induction, there exist edge-disjoint paths joining the terminals $T^{\prime}$ in $H^{\prime}$; together with the one-edge path from $v$ to $w$, this yields the desired paths joining $T$ in $H$.

(ii) $w \notin T$. In this case, we again define $H^{\prime}$ to be the tree $H$ with edge $(v, w)$ and node $v$ deleted, and define $T^{\prime \prime}=(T \backslash\{v\}) \cup\{w\}$. By induction, there exist edge-disjoint paths joining the terminals $T^{\prime \prime}$ in $H^{\prime}$; by concatenating the edge $(v, w)$ to the end of the path terminating at $w$, we obtain the desired paths joining $T$ in $H$.

Lemma 2.5. The $V C$-dimension of $(V, \mathcal{S})$ is at most $2 k+1$.

Proof. Let us consider an arbitrary set $A \subseteq V$ of size $2 k+2$; we must show that it is not shattered by $(V, \mathcal{S})$. We apply Lemma 2.4 , obtaining a set of mutually edge-disjoint paths $P_{1}, P_{2}, \ldots, P_{k+1}$ such that each node in $A$ appears as one end of exactly one of these paths. Relabeling $A$ if necessary, we will assume that $P_{i}$ has ends equal to $a_{i}$ and $a_{i+k+1}$.

Now we claim that $B=\left\{a_{1}, a_{2}, \ldots, a_{k+1}\right\}$ cannot be written as $A \cap S$ for any $k$-edge-separable set $S$. Suppose it could, and let $Z \subseteq E$ have the property that $|Z| \leq k$ and $S$ is a union of connected components of $G \backslash Z$. Since $|Z|<k+1$, there is some path $P_{i}$ that contains no edge in $Z$. But then we have $a_{i} \in S$, $a_{i+k+1} \notin S$, and a path connecting them in $G \backslash Z$-this is a contradiction.

By Lemma 2.5 and Theorem 2.3, a small random set of nodes of $V$ will form an $\varepsilon$-net of the set system $(V, \mathcal{S})$ with high probability. By Lemma 2.1, such a set system will be a detection set. Thus we have the following theorem.

Theorem 2.6. A random subset of $V$ of size

$$
f(2 k+1, \varepsilon, \delta)=O\left(k \varepsilon^{-1} \log \varepsilon^{-1}+\varepsilon^{-1} \log \delta^{-1}\right)
$$


is an $(\varepsilon, k)$-detection set with respect to edge failures with probability at least $1-\delta$.

In fact, the bound on the size of a random subset needed in Theorem 2.6 is asymptotically tight. Consider a graph $G$ that is a two-level rooted tree; the root $v$ has a set of $c$ children $W=\left\{w_{1}, \ldots, w_{c}\right\}$, where $c=\Omega\left(k \varepsilon^{-1}\right)$, and each node $w_{i}$ has at least $\varepsilon n / k$ children. Now, standard bounds for occupany problems [Alon and Spencer 92] show that if we choose a random sample $D$ of size $o\left(k \varepsilon^{-1} \log \varepsilon^{-1}\right)$, then with high probability, $D$ fails to intersect at least $k$ of the subtrees rooted at nodes in $W$. Let $F$ denote the set of edges from $v$ to $k$ of these nodes in $W$; we observe that $F$ is an $(\varepsilon, k)$-partitioning set, and all nodes in $D$ belong to a single component of $G \backslash F$.

For the case in which only edges can fail, our notion of detection sets is equivalent to Gupta's notion of weak detection sets [Gupta 00]. As a result, his theorem shows that every graph has an $(\varepsilon, k)$-detection set with respect to edge failures of size $O\left(k \varepsilon^{-1}\right)$. (The example of the previous paragraph shows that there are graphs in which a detection set of such a size cannot be obtained through uniform random sampling.)

We conclude this section with a lower bound matching the result in Lemma 2.5.

Proposition 2.7. There exist graphs $G=(V, E)$ for which the $V C$-dimension of the associated set system $(V, \mathcal{S})$ is equal to $2 k+1$.

Proof. Since Lemma 2.5 provides an upper bound, we need only demonstrate a graph $G=(V, E)$ for which some set of $2 k+1$ nodes is shattered by $k$-edgeseparable sets.

Let $G$ be the star graph $K_{1,2 k+1}$, and let $A$ be the leaves of $G$; we claim that $A$ is shattered by $(V, \mathcal{S})$. Indeed, let $B$ be any subset of $A$. If $|B| \leq k$, then $B$ is $k$-edge-separable as we may delete the set $Z$ of all edges incident to nodes in $B$. Hence, $B=A \cap S$ for a set $S \in \mathcal{S}$. On the other hand, if $|B|>k$, then $|A \backslash B| \leq k$. In this case, we may delete the set $Z$ of all edges incident to nodes in $A \backslash B$; one of the components of the resulting graph is $V \backslash(A \backslash B)$, and $B=A \cap(V \backslash(A \backslash B))$.

\section{Detecting General Failures}

Analyzing the construction of detection sets when both nodes and edges can fail is a much more complicated problem. To see some of the issues involved, we first consider what goes wrong when we try to extend the technique of the previous section in a direct way. 
By analogy with the notion of $k$-edge-separability, we can define a set $S$ to be $k$-separable if there exists a set $Z$ of at most $k$ elements (i.e. nodes or edges) such that $S$ is the union of connected components of $G \backslash Z$. Now, a direct analogue of Lemma 2.1 still applies with this new definition: an $\varepsilon$-net with respect to $k$-separable sets would indeed be an $(\varepsilon, k)$-detection set. Unfortunately, the corresponding analogue of Lemma 2.5 now fails badly: The VC-dimension of the $k$-separable sets in a graph can be nearly as large as the size of the vertex set itself. Consider the star graph $K_{1, n-1}$ : Every subset of the leaves is 1-separable, as can be seen by deleting the singleton set $Z$ consisting of the "center" of the star. Consequently, for this graph, the approach of the previous section will yield an upper bound for the size of $(\varepsilon, k)$-detection sets that is actually larger than $n$, the number of nodes.

However, while the star graph shows that $k$-separable sets can have large VC-dimension, it does not provide a counterexample to the existence of small detection sets. We simply need to base our analysis on something stronger than $k$-separable sets. Thus, in this section, we consider a more complicated set system defined over the vertex set $V$ of a graph, establish a bound on the VC-dimension of this set system, and show that $\varepsilon$-nets for this set system yield detection sets.

We begin with a connected graph $G=(V, E)$, and a partition of $V$ into sets $V_{0}$ of end nodes and $V_{1}$ of internal nodes. Recall that our detection set $D$ must be a subset of $V_{0}$ (i.e., we can only place monitoring agents at end nodes), and that we are trying to detect partitions of $V_{0}$. Let $n_{0}=\left|V_{0}\right|$ and $n_{1}=\left|V_{1}\right|$. We will assume that $k<\frac{1}{3} \varepsilon n_{0}$; for otherwise, we can choose all of $V_{0}$ as our detection set and still remain within the bounds of Theorem 1.2.

We first subdivide each edge of $G$, adding each of the subdividing nodes to the set $V_{1}$. In this way, we may assume that any $(\varepsilon, k)$-partitioning set $Z$ we consider consists only of nodes; for if it contains an edge $e$, we can replace $e$ with an appropriate subdividing node in $V_{1}$, and this new set will induce the same partition of $V_{0}$.

Now, what was the underlying problem in using the collection of $k$-separable sets? Essentially, it allowed too many possible unions of distinct components. We need a way to reduce the combinatorial complexity of the underlying set system, by restricting the way in which we form unions of components, and at the same time keep the set system rich enough that its $\varepsilon$-nets form detection sets. One natural way is to impose a lexicographic order on nodes, and hence on components, and force our unions to respect this ordering. Thus, let us arbitrarily index the vertex set $V=\left\{v_{1}, v_{2}, \ldots, v_{n}\right\}$. Given two disjoint subsets $A$ and $B$, we say that $A$ precedes $B$ lexicographically if the minimum index of a node in $A$ is less than the minimum index of a node in $B$. Now, we say that $S$ is a $k$-segmental set if it can be obtained in the following way: 
(i) Choose a set $Z$ of at most $k$ nodes. (Recall that we will only consider the deletion of nodes from now on.)

(ii) Let $U_{1}, U_{2}, \ldots, U_{s}$ be the connected components of $G \backslash Z$, indexed in ascending lexicographic order.

(iii) For numbers $p$ and $q(1 \leq p \leq q \leq s)$, define $S=U_{p} \cup U_{p+1} \cup \cdots \cup U_{q}$.

Thus, briefly, $S$ is the union of a set of connected components of $G \backslash Z$ that are consecutive in lexicographic order. We will say that $Z$ is the generator of $S$, and that $\left\{U_{p}, \ldots, U_{q}\right\}$ are the constituents of $S$.

We first establish that the set system of $k$-segmental sets is related to the construction of detection sets. For a subset of nodes $U$, we define its $V_{0}$-weight to be the cardinality of $U \cap V_{0}$, and we denote this quantity by $\pi_{0}(U)=\left|U \cap V_{0}\right|$.

Lemma 3.I. If $D \subseteq V$ intersects every $k$-segmental set of $V_{0}$-weight at least $\frac{1}{3} \varepsilon n_{0}$, then it is an $(\varepsilon, k)$-detection set for $V_{0}$.

Proof. Let $Z$ be a set of nodes of size $k^{\prime} \leq k$, and let $U_{1}, \ldots, U_{s}$ be the components of $G \backslash Z$ in ascending lexicographic order. Suppose there exist disjoint sets $A, B \subseteq V \backslash Z$, each of $V_{0}$-weight at least $\varepsilon n_{0}$, that are separated in $G \backslash Z$. In this case, it follows that no component $U_{i}$ has $V_{0}$-weight greater than $(1-\varepsilon) n_{0}$.

Now, let $j$ be the minimum index such that $\pi_{0}\left(U_{1} \cup \cdots \cup U_{j}\right)>\frac{1}{3} \varepsilon n_{0}$; then we must have

$$
\begin{aligned}
\pi_{0}\left(U_{1} \cup \cdots \cup U_{j}\right) & \leq \frac{1}{3} \varepsilon n_{0}+(1-\varepsilon) n_{0} \\
& \leq\left(1-\frac{2}{3} \varepsilon\right) n_{0} .
\end{aligned}
$$

Thus,

$$
\begin{aligned}
\pi_{0}\left(U_{j+1} \cup \cdots \cup U_{s}\right) & \geq n_{0}-k^{\prime}-\left(1-\frac{2}{3} \varepsilon\right) n_{0} \\
& =\frac{2}{3} \varepsilon n_{0}-k^{\prime} \geq \frac{1}{3} \varepsilon n_{0} .
\end{aligned}
$$

Now, $U_{1} \cup \cdots \cup U_{j}$ and $U_{j+1} \cup \cdots \cup U_{s}$ are both $k$-segmental sets of $V_{0}$-weight at least $\frac{1}{3} \varepsilon n_{0}$, and so $D$ contains at least one node in each; and these lie in different components of $G \backslash Z$, as required.

Let $\mathcal{E}$ denote the collection of all $k$-segmental subsets of $V$. We wish to show that $(V, \mathcal{E})$ has bounded VC-dimension; then Lemma 2.2 will imply that $\left(V_{0}, \mathcal{E} \mid V_{0}\right)$ has bounded VC-dimension as well, allowing us to use Theorem 2.3 in conjunction 
with Lemma 3.1. In the analogous situation in the previous section, we used an argument based on the fact that every subset of nodes of even cardinality could be partitioned into two subsets linked by edge-disjoint paths. But this approach cannot be directly applied here: In the example of $K_{1, n-1}$, we see that a graph can have a large set of nodes (the leaves) for which no two large disjoint subsets can be paired up via node-disjoint paths.

Thus, linkages via node-disjoint paths behave very differently from linkages via edge-disjoint paths. To analyze the obstacles to linkages via node-disjoint paths, we use the following min-max theorem due to Gallai [Gallai 61]

Theorem 3.2. ([Gallai 61]) Let $H=\left(V_{H}, E_{H}\right)$ be a graph, and let $A \subseteq V_{H}$. Suppose that for a number $\ell$, there is no set of $\ell+1$ mutually node-disjoint paths in $H$, all of whose ends are in $A$. Then there exists a set $W \subseteq V_{H}$ so that $G \backslash W$ has components $X_{1}, \ldots, X_{t}$ and

$$
|W|+\sum_{i=1}^{t}\left\lfloor\frac{\left|A \cap X_{i}\right|}{2}\right\rfloor \leq \ell .
$$

Note that $W$ constitutes a witness to the fact that no set of $\ell+1$ disjoint paths exists: Each path with ends in $A$ must "consume" a node in $W$ (accounted for by the first term) or two nodes of $A$ that lie in the same component of $G \backslash W$ (accounted for by the second term). We refer the reader to [Frank 90, Schrijver 01] for a discussion of Theorem 3.2 and some related results, including a deep generalization due to Mader [Mader 78].

As a first step, we derive a consequence of Theorem 3.2 that is slightly weaker, but easier to use in the arguments to follow.

Lemma 3.3. Let $H=\left(V_{H}, E_{H}\right)$ be a graph, and let $A \subseteq V_{H}$. Suppose that for a number $\ell$, there is no set of $\ell+1$ mutually node-disjoint paths in $H$, all of whose ends are in $A$. Then there is a set $W^{\prime} \subseteq V_{H}$ of size at most $3 \ell$ such that each node in $A \backslash W^{\prime}$ belongs to a different component of $H \backslash W^{\prime}$.

Proof. By Theorem 3.2, there is a set $W \subseteq V_{H}$ so that $G \backslash W$ has components $X_{1}, \ldots, X_{t}$ and

$$
|W|+\sum_{i=1}^{t}\left\lfloor\frac{\left|A \cap X_{i}\right|}{2}\right\rfloor \leq \ell .
$$

We say that a component $X_{i}$ is large if $\left|A \cap X_{i}\right| \geq 2$, and we let $W^{*}$ denote the set of all nodes in $A$ that belong to large components $X_{i}$. In other words,

$$
W^{*}=\bigcup_{X_{i} \text { large }}^{\bigcup}\left(A \cap X_{i}\right) .
$$


Since each large component $X_{i}$ contributes at least 1 to the left-hand side of the above inequality, there can be at most $\ell$ large components. Hence,

$$
\begin{aligned}
\left|W \cup W^{*}\right| & =|W|+\sum_{X_{i} \text { large }}\left|A \cap X_{i}\right| \\
& \leq|W|+\left[\sum_{X_{i} \text { large }} 1+2\left\lfloor\frac{\left|A \cap X_{i}\right|}{2}\right]\right] \\
& \leq \ell+2\left[|W|+\sum_{i=1}^{t}\left\lfloor\frac{\left|A \cap X_{i}\right|}{2}\right\rfloor\right] \\
& \leq 3 \ell .
\end{aligned}
$$

Finally, we define $W^{\prime}=W \cup W^{*}$ and claim that no two nodes of $A \backslash W^{\prime}$ belong to the same component of $H \backslash W^{\prime}$. Indeed, if $a_{i}, a_{j} \in A$ were to belong to the same component of $H \backslash W^{\prime}$, then they would also belong to the same component $X_{i}$ of $H \backslash W$; but then $X_{i}$ would be a large component, and we would have $a_{i}, a_{j} \in W^{*} \subseteq W^{\prime}$, a contradiction.

We now analyze the VC-dimension of $(V, \mathcal{E})$ using a two-pronged argument: For any set $A$ of sufficient cardinality, either $G$ contains many mutually node-disjoint paths with ends in $A$, or it contains a disconnecting set $W^{\prime}$ as in Lemma 3.3. In both cases, we argue that $A$ cannot be shattered.

Lemma 3.4. The $V C$-dimension of $(V, \mathcal{E})$ is at most $O\left(k^{3}\right)$.

Proof. Recall that we have fixed a lexicographic ordering of $V$, as $v_{1}, v_{2}, \ldots, v_{n}$. We define $r=3(3 k+1)(k+1)^{2}+3 k$ and suppose by way of contradiction that there is a set $A$, of size greater than $r$, that is shattered by $(V, \mathcal{E})$.

We first claim:

(i) There is no set of a $k+1$ mutually node-disjoint paths, all of whose ends are in $A$.

Suppose there were such paths $P_{1}, \ldots, P_{k+1}$, such that $P_{i}$ has ends $a_{i}, a_{i}^{\prime} \in A$. Now, by our assumption that $A$ is shattered, there is a $k$-segmental set $S$ such that $B=\left\{a_{1}, \ldots, a_{k+1}\right\}=A \cap S$. Let $Z$ be a generator of $S$. Then since $|Z|<k+1$, there is some path $P_{i}$ that contains no node in $Z$. But this is a contradiction, since $a_{i} \in S, a_{i}^{\prime} \notin S$, and $P_{i}$ is a path connecting them in $G \backslash Z$.

By (i), and Lemma 3.3, there is a set $W^{\prime}$ of size at most $3 k$ such that every node in $A \backslash W^{\prime}$ belongs to a different component of $G \backslash W^{\prime}$. For a node $v \in V \backslash W^{\prime}$, we use $C(v)$ to denote the component of $G \backslash W^{\prime}$ containing $v$, and $N(v)$ to denote the set of nodes in $W^{\prime}$ that are neighbors of at least one node in $C(v)$. We write 
$A^{\prime}=A \backslash W^{\prime}=\left\{a_{1}^{\prime}, \ldots, a_{s}^{\prime}\right\}$, where $s \geq r-3 k$ and the indexing need not be related to the lexicographic order on $V$.

The remainder of the proof is guided by the following basic idea. We will consider a subset of $A^{\prime}$ (called $B^{\prime \prime}$ below) such that the components of $G \backslash W^{\prime}$ containing nodes of $B^{\prime \prime}$ are "interlaced" in lexicographic order with components containing nodes of $A^{\prime} \backslash B^{\prime \prime}$. Now, for any supposed $k$-segmental set $S$ so that $A \cap S=B^{\prime \prime}$, we will show that several of the components of $G \backslash W^{\prime}$ are, in fact, constituents of $S$. Because of the lexicographic interlacing of the components of $G \backslash W^{\prime}$, and because $S$ must consist of components that are contiguous in lexicographic order, we will find that in order to include all nodes of $B^{\prime \prime}, S$ must include at least one node from $A^{\prime} \backslash B^{\prime \prime}$. This will contradict our assumption that $A \cap S=B^{\prime \prime}$, and hence show that $A$ cannot be shattered.

We define a parameter $\gamma=3(k+1)^{2}$. We define the weight $w_{v}$ of a node $v \in W^{\prime}$ to be the number of sets $N\left(a_{i}^{\prime}\right), a_{i}^{\prime} \in A^{\prime}$, that contain $v$. We say $v \in W^{\prime}$ is heavy if $w_{v} \geq \gamma$; otherwise, we say it is light. Let $W_{h}$ denote the heavy nodes in $W^{\prime}$, and $W_{\ell}$ denote the light nodes. We say a node $a_{i}^{\prime} \in A^{\prime}$ is typical with respect to $W_{h}$ and $W_{\ell}$ if $N\left(a_{i}^{\prime}\right) \subseteq W_{h}$; otherwise, we say it is atypical.

Consider performing the following procedure. We delete all the sets $C\left(a_{i}^{\prime}\right)$ for which $a_{i}^{\prime}$ is atypical; update $A^{\prime}$, the weights $\left\{w_{v}: v \in W^{\prime}\right\}$, and the sets $W_{h}$ and $W_{\ell} ;$ and then iterate. We stop when there are no atypical nodes remaining. We claim:

(ii) At most $3 k \gamma$ nodes of $A^{\prime}$ are deleted by the above procedure.

We prove this by the following counting argument. First, observe that for any node $v \in W^{\prime}$, there is at most one iteration in which it is light, but has positive weight. Indeed, the weight of $v$ never increases; and in the first iteration in which it is declared light, all sets $C\left(a_{i}^{\prime}\right)$ for which $v \in N\left(a_{i}^{\prime}\right)$ are deleted, and so the weight of $v$ is reduced to zero. Now, consider any node $a_{i}^{\prime} \in A^{\prime}$ that is deleted. In the iteration in which it is deleted, there is some $v \in W_{\ell} \cap N\left(a_{i}^{\prime}\right)$; we charge $a_{i}^{\prime}$ to $v$. A node $v \in W^{\prime}$ can only be charged in the single iteration when it is light but has positive weight; in this iteration, at most $\gamma$ nodes in $A^{\prime}$ can be charged to it. Since $\left|W^{\prime}\right| \leq 3 k$, (ii) follows.

Thus, when this deletion procedure terminates, we have a final partition of $W^{\prime}$ into heavy and light nodes, which we will refer to as $W_{h}$ and $W_{\ell}$ for the remainder of the proof. We define $A^{\prime \prime} \subseteq A^{\prime}$ to be the set of nodes not deleted from $A^{\prime}$ by the deletion procedure; by (ii), we know that there are at least $s-3 k \gamma$ such nodes. We also know that all nodes in $A^{\prime \prime}$ are typical with respect to $W_{h}$ and $W_{\ell}$, and that for each node $v \in W_{h}$, there are at least $\gamma$ nodes $a^{\prime \prime} \in A^{\prime \prime}$ for which $v \in N\left(a^{\prime \prime}\right)$. 
We now partition $A^{\prime \prime}$ into sets $B$ and $B^{\prime}$ as follows. For each node $v \in W_{h}$, we select nodes $b_{v, 1}, \ldots, b_{v, k+1} \in A^{\prime \prime}$ with the property that $v \in N\left(b_{v, i}\right)$, and place them in $B$. We define $B^{\prime}=A^{\prime \prime} \backslash B$. Since $|B| \leq 3 k(k+1),\left|B^{\prime}\right|=t \geq$ $s-3 k \gamma-3 k(k+1)=3(k+1)$. We index $B^{\prime}=\left\{b_{1}^{\prime}, b_{2}^{\prime}, \ldots, b_{t}^{\prime}\right\}$ in ascending lexicographic order of the sets $\left\{C\left(b_{i}^{\prime}\right)\right\}$.

Now, partition $B^{\prime}$ into $B_{1}^{\prime}=\left\{b_{1}^{\prime}, b_{2}^{\prime}, \ldots, b_{k+1}^{\prime}\right\}, B_{2}^{\prime}=\left\{b_{k+2}^{\prime}, \ldots, b_{2 k+2}^{\prime}\right\}$, and $B_{3}^{\prime}=\left\{b_{2 k+3}^{\prime}, \ldots, b_{t}^{\prime}\right\}$. Note that each of $B_{1}^{\prime}, B_{2}^{\prime}$, and $B_{3}^{\prime}$ has at least $k+1$ elements. We define $B^{\prime \prime}=B_{1}^{\prime} \cup B_{3}^{\prime}$, and claim:

(iii) There is no k-segmental set $S \subseteq V$ so that $B^{\prime \prime}=A \cap S$.

This will yield a contradiction to our assumption that $A$ is shattered, and hence prove the lemma.

To prove (iii), suppose there were such a set $S$, with generator $Z$. Let $U_{1}, U_{2}, \ldots, U_{s}$ denote the components of $G \backslash Z$ in lexicographic order; for some indices $p$ and $q$, we have $S=U_{p} \cup U_{p+1} \cup \cdots \cup U_{q}$. First we claim:

(iii(a)) Each node of $B^{\prime \prime}$ belongs to a different constituent of $S$.

Suppose not, and let $b_{i}^{\prime}, b_{j}^{\prime} \in B^{\prime \prime}$ belong to the same constituent $U_{\ell}$. Then there is a path $P \subseteq U_{\ell}$ with ends equal to $b_{i}^{\prime}$ and $b_{j}^{\prime}$. Since $b_{i}^{\prime}$ and $b_{j}^{\prime}$ belong to distinct components of $G \backslash W^{\prime}$, there is a node $v \in W^{\prime} \cap P$, and hence in $W^{\prime} \cap U_{\ell}$. Now consider the nodes $b_{v, 1}, \ldots, b_{v, k+1}$ that were placed in $B$ because $v \in N\left(b_{v, i}\right)$ for $i=1,2, \ldots, k+1$. For each such node $b_{v, i}$, there is a path $Q_{i}$ with ends equal to $v$ and $b_{v, i}$ such that $W^{\prime} \cap Q_{i}=\{v\}$. But $v \notin Z$, and $|Z|<k+1$; thus, there is some $Q_{i}$ that does not meet $Z$. It follows that the corresponding node $b_{v, i}$ belongs to $U_{\ell}$, and hence, belongs to $S$. But $b_{v, i} \notin B^{\prime \prime}$, contradicting our assumption that $B^{\prime \prime}=A \cap S$. This proves (iii(a)).

Next, we claim:

(iii(b)) $W_{h} \subseteq Z$.

Again, suppose not, and say that $v \in W_{h} \backslash Z$. Now, $A^{\prime \prime}$ contains at least $\gamma$ nodes $a^{\prime \prime}$ for which $v \in N\left(a^{\prime \prime}\right)$. We have $|B| \leq 3 k(k+1)$ and $\left|B_{2}^{\prime}\right|=k+1$; thus $B^{\prime \prime}=A^{\prime \prime} \backslash\left(B \cup B_{2}^{\prime}\right)$ contains at least $\gamma-(3 k+1)(k+1) \geq k+2$ nodes $b^{\prime \prime}$ for which $v \in N\left(b^{\prime \prime}\right)$. Let $B_{v}^{\prime \prime}$ denote this set of nodes in $B^{\prime \prime}$. Since $|Z| \leq\left|B_{v}^{\prime \prime}\right|-2$, there exist two nodes $b_{v}^{\prime}, b_{v}^{\prime \prime} \in B_{v}^{\prime \prime}$ such that $Z \cap\left(C\left(b_{v}^{\prime}\right) \cup C\left(b_{v}^{\prime \prime}\right)\right)=\phi$. But this implies there is a path in $G \backslash Z$ with ends equal to $b_{v}^{\prime}$ and $b_{v}^{\prime \prime}$, contradicting (iii(a)); this proves (iii(b))

Since $A^{\prime \prime}$ consists entirely of typical nodes, the following is a direct consequence of (iii(b)).

(iii(c)) For each $a^{\prime \prime} \in A^{\prime \prime}$, we have $N\left(a^{\prime \prime}\right) \subseteq Z$.

Now, since $B_{1}^{\prime}, B_{2}^{\prime}$, and $B_{3}^{\prime}$ each have at least $k+1$ nodes, there exist nodes $b_{i}^{\prime} \in B_{1}^{\prime}, b_{j}^{\prime} \in B_{2}^{\prime}$, and $b_{\ell}^{\prime} \in B_{3}^{\prime}$ such that $Z \cap\left(C\left(b_{i}^{\prime}\right) \cup C\left(b_{j}^{\prime}\right) \cup C\left(b_{k}^{\prime}\right)\right)=\phi$. 
Combined with (iii (c)), this implies

(iii(d)) Each of $C\left(b_{i}^{\prime}\right), C\left(b_{j}^{\prime}\right)$, and $C\left(b_{\ell}^{\prime}\right)$ is a component of $G \backslash Z$.

Finally, recall that we indexed $B^{\prime}=\left\{b_{1}^{\prime}, b_{2}^{\prime}, \ldots, b_{t}^{\prime}\right\}$ in ascending lexicographic order of the sets $C\left(b_{1}^{\prime}\right), \ldots, C\left(b_{t}^{\prime}\right)$. But since $b_{i}^{\prime}, b_{\ell}^{\prime} \in B^{\prime \prime}$, (iii(d)) implies that $C\left(b_{i}^{\prime}\right)$ and $C\left(b_{\ell}^{\prime}\right)$ are constituents of $S$. Now, $C\left(b_{j}^{\prime}\right)$ is a component of $G \backslash Z$ that comes between $C\left(b_{i}^{\prime}\right)$ and $C\left(b_{\ell}^{\prime}\right)$ lexicographically; since $S$ is $k$-segmental, it follows that $C\left(b_{j}^{\prime}\right) \subseteq S$ and hence $b_{j}^{\prime} \in S$. But $b_{j}^{\prime} \notin B^{\prime \prime}$, contradicting our assumption that $B^{\prime \prime}=A \cap S$. This contradiction establishes (iii), and hence the lemma.

Finally, we prove the theorem described initially.

Theorem I.2 For every graph $G=(V, E)$, and any set $V_{0} \subseteq V$, there is an $(\varepsilon, k)$ detection set for $V_{0}$ of size $O\left(k^{3} \varepsilon^{-1} \log \varepsilon^{-1}\right)$. Moreover, a set of

$$
O\left(k^{3} \varepsilon^{-1} \log \varepsilon^{-1}+\varepsilon^{-1} \log \delta^{-1}\right)
$$

nodes chosen uniformly at random from $V_{0}$ is an $(\varepsilon, k)$-detection set for $V_{0}$ with probability at least $1-\delta$.

Proof. By Lemmas 2.2 and 3.4, the VC-dimension of $\left(V_{0}, \mathcal{E} \mid V_{0}\right)$ is $O\left(k^{3}\right)$. Thus, by Theorem 2.3, a random set of $O\left(k^{3} \varepsilon^{-1} \log \varepsilon^{-1}+\varepsilon^{-1} \log \delta^{-1}\right)$ nodes chosen uniformly at random from $V_{0}$ is a $\frac{1}{3} \varepsilon$-net for this set system with probability at least $1-\delta$.

By definition, such a $\frac{1}{3} \varepsilon$-net $D$ has the property that for any $S \in \mathcal{E}$, if $\left|S \cap V_{0}\right| \geq$ $\frac{1}{3} \varepsilon n_{0}$, then $S \cap V_{0}$ contains an element of $D$. Thus, $D$ intersects every $k$-segmental set of $V_{0}$-weight at least $\frac{1}{3} \varepsilon n_{0}$, and so by Lemma 3.1 , it is an $(\varepsilon, k)$-detection set for $V_{0}$.

\section{Further Extensions: Probabilistic Adversaries}

Using our analysis of detection sets, we can extend our results to a setting in which nodes and edges can fail according to an unknown (adversarially chosen) probability distribution. Specifically, we consider a model in which an adversary assigns a failure probability $p_{j}$ to each element $j$ of $G$, subject only to the condition that the mean number of failures $\sum_{j \in V \cup E} p_{j}$ is bounded by a number $k$. Note that since an adversary could assign a failure probability of 1 to $k$ elements, and 0 to all others, this model clearly includes our initial adversarial model. 
Under this model, we can show that a random subset $D$ of $V_{0}$ of size $O\left(k^{3} \varepsilon^{-1} \delta^{-3} \log \left(k \varepsilon^{-1} \delta^{-1}\right)\right)$ has the following property with high probability: If elements fail according to any assignment of probabilities $\left\{p_{j}\right\}$ as above, the probability that two subsets of $V_{0}$ of size $\varepsilon\left|V_{0}\right|$ become separated but all nodes of $D$ remain connected is at most $\delta$. In other words, regardless of the distribution of failures in this model (assuming only that they have bounded mean), there is only a small probability that our set $D$ will fail to "witness" any significant partition of the end nodes.

The proof of this statement is a direct consequence of Markov's inequality combined with Theorem 1.2. Indeed, let $\mathcal{B}$ denote the event that more than $k \delta^{-1}$ elements of $G$ are deleted; let $\mathcal{E}$ denote the event that two subsets of $V_{0}$ of size $\varepsilon\left|V_{0}\right|$ become separated by the deletion process; and let $\mathcal{F}$ denote the event that all nodes of $D$ lie in a single component of $G$ after the deletion occurs. We want to show that $\operatorname{Pr}[\mathcal{E} \cap \mathcal{F}] \leq \delta$. But if the event $\mathcal{B}$ does not occur, then Theorem 1.2 implies $D$ is large enough so that the event $\mathcal{E} \cap \mathcal{F}$ cannot occur; hence we have $\mathcal{E} \cap \mathcal{F} \subseteq \mathcal{B}$. By Markov's inequality, $\operatorname{Pr}[\mathcal{B}] \leq \delta$, since the expected number of elements deleted is at most $k$; it follows that $\operatorname{Pr}[\mathcal{E} \cap \mathcal{F}] \leq \operatorname{Pr}[\mathcal{B}] \leq \delta$.

Acknowledgements. I thank the anonymous referees for suggestions that helped to improve the presentation. A preliminary version of this paper appeared in the Proceedings of the 41st IEEE Symposium on Foundations of Computer Science, 2000. This work was supported in part by a David and Lucile Packard Foundation Fellowship, an ONR Young Investigator Award, and NSF Faculty Early Career Development Award CCR9701399.

\section{References}

[Alon and Spencer 92] N. Alon and J. Spencer. The Probabilistic Method. New York: John Wiley and Sons, 1992.

[Berge 58] C. Berge, "Sur le couplage maximum d'un graphe," C.R. Acad. Sci. Paris 247 (1958), 258-259.

[Bharat and Broder 98] K. Bharat and A. Broder. "A Technique for Measuring the Relative Size and Overlap of Public Web Search Engines." In Proc. 7th Intl. WWW Conference, pp. 379-388, Amsterdam: Elsevier Science, 1998.

[Blumer 89] A. Blumer, A. Ehrenfeucht, D. Haussler and M. Warmuth. "Learnability and the Vapnik-Chervonenkis Dimension." J. of the ACM 36:(4) (1989), 929-965.

[Chung et al. 01] F. Chung, M. Garrett, R. Graham, and D. Shallcross. "Distance Realization Problems with Applications to Internet Tomography." Journal of Computer and System Sciences 63 (2001), 432-448. 
[Claffy et al. 99] K. Claffy, T. Monk, and D. McRobb. "Internet Tomography." Nature. Available from World Wide Web (http://nature.com/nature/webmatters/tomog/ tomog.html), 1999.

[Fakcharoenphol 01] Jittat Fakcharoenphol. "An Improved VC-Dimension Bound for Finding Network Failures." Master's thesis, U.C. Berkeley, 2001.

[Frank 90] A. Frank. "Packing Paths, Cuts, and Circuits-A Survey." In Paths, Flows, and VLSI-Layout, edited by B. Korte, L. Lovász, H.J. Prömel, and A. Schrijver, pp. 49-100. Berlin: Springer-Verlag, 1990.

[Gallai 61] T. Gallai. "Maximum-minimum Sätze und verallgemeinerte Faktoren von Graphen." Acta Math. Acad. Sci. Hungaricae 12 (1961), 131-173.

[Gupta 00] A. Gupta. November 2000, described in Section 1.4.2 of [Fakcharoenphol $01]$.

[Haussler and Welzl 87] D. Haussler, E. Welzl, " $\varepsilon$-nets and Simplex Range Queries." Discrete and Computational Geom. 2 (1987), 127-151.

[Karger 95] D. Karger. "A Randomized Fully Polynomial Time Approximation Scheme for the All-Terminal Network Reliability Problem." In Proc. ACM Symp. on Theory of Computing, pp. 11-17. New York: ACM Press, 1995.

[Keynote 03] Keynote Systems. Available from World Wide Web (http://www. keynote.com), 2003.

[Lawrence and Giles 99] S. Lawrence and C.L. Giles. "Accessibility of Information on the Web." Nature 400 (1999), 107-109.

[Lovász and Plummer] L. Lovász and M. Plummer. Matching Theory. Amsterdam: North-Holland, 1986.

[Mader 78] W. Mader. "Über die Maximalzahl kreuzungsfreier H-Wege." Arch. Math. 31 (1978), 387-402.

[Mahdavi and Paxson 99] J. Mahdavi and V. Paxson. "IPPM Metrics for Measuring Connectivity." RFC 2678, Network Working Group, Sept. 1999.

[Paxson 96] V. Paxson. "Towards a Framework for Defining Internet Performance Metrics." In Proc. Internet Society (ISOC) INET Conference. Available from World Wide Web (http://www.isoc.org/inet96/proceedings/longtoc.htm), Section D3, 1996.

[Ratnasamy and McCanne 99] S. Ratnasamy and S. McCanne. "Inference of Multicast Routing Trees and Bottleneck Bandwidths using End-to-end Measurements." In Proc. IEEE INFOCOM, pp. 353-360. Los Alamitos, CA: IEEE Press, 1999.

[Schrijver 01] A. Schrijver. "A Short Proof of Mader's $\mathcal{S}$-Paths Theorem." Journal of Combinatorial Theory Series B 82 (2001), 319-321.

[Siamwalla et al. 98] R. Siamwalla, R. Sharma, and S. Keshav. "Discovering Internet Topology." Available from World Wide Web (http://www.cs.cornell.edu/ skeshav/papers.html), 1998.

[Vapnik and Chervonenkis 71] V. N. Vapnik and A. Y. Chervonenkis. "On the Uniform Convergence of Relative Frequencies of Events to their Probabilities." Theory of Prob. App. 16 (1971), 264-280. 
Jon Kleinberg, Department of Computer Science, Cornell University, Ithaca, NY 14853 (kleinber@cs.cornell.edu)

Received October 31, 2002; accepted December 11, 2002 
